\title{
Les Fractures De La Patella Chez L'adulte
}

\author{
Gnandi-Piou F.,
}

Service de Traumatologie Orthopédie, CHU Kara, Kara, Togo

Akondo HK F.,

Service de Traumatologie Orthopédie, CHU Sylvanus Olympio, Lomé, Togo

\author{
Lagnéblé A., \\ Tsolenyanu S.,
}

Service de Traumatologie Orthopédie, CHU Campus, Lomé - Togo

Amakoutou K.,

Service de Traumatologie Orthopédie, CHU Kara, Kara, Togo

Walla A.,

Service de Traumatologie Orthopédie, CHU Campus, Lomé - Togo

\section{Abalo A.,}

Service de Traumatologie Orthopédie, CHU Sylvanus Olympio, Lomé, Togo

\section{Dossim AM.,}

Service de Traumatologie Orthopédie, CHU Kara, Kara, Togo

Service de Traumatologie Orthopédie, CHU Sylvanus Olympio, Lomé, Togo

Service de Traumatologie Orthopédie, CHU Campus, Lomé - Togo

Doi: 10.19044/esj.2018.v14n36p37 URL:http://dx.doi.org/10.19044/esj.2018.v14n36p37

\begin{abstract}
Objectives: to determine the epidemiological aspects, the different techniques of surgical fixation of the patella fractures and the results. Patients and methods: this was a retrospective study from January 1, 2013 to December 31, 2017 and included the records of patients aged 15 years and older surgically treated for a patella fracture in our center. Results: The work involved 36 patients including 28 men (77.8\%) and 8 women $(22.2 \%)$ with a mean age of 35.9 years. High way trauma were the first cause of these fractures. Fractures were open in $44.4 \%$ of cases. Type II according to Ricard and Moulay classification was the most represented with $58.3 \%$ of cases. The majority of the patients, $80.5 \%$, were treated by rigging. Five (5) cases of hematoma, one (1) postoperative infection, and three (3) cases of knee stiffness complicated the short- and medium-term follow-up. With an average follow-up of 2 years 6 months, radiologically, normal consolidation was achieved in $86.1 \%$ of cases. Two (2) cases of delayed consolidation and three (3) cases of nonunion have been noted. Three (3) cases of patellofemoral
\end{abstract}


osteoarthritis appeared at two years. From the functional point of view, according to the Bosman score, $27.8 \%$ were excellent, $63.9 \%$ were good, $8.3 \%$ were bad, and the cable rigging seemed to give excellent and good results. Conclusion: the treatment of transverse fractures of the patella must be exclusively surgical. Guying is an osteosynthesis technique of choice. Nevertheless complications including knee stiffness, non-union and patellofemoral osteoarthritis are not uncommon.

Keywords: Patella fracture, rigging, stiffness, osteoarthritis

\section{Resume}

Objectifs : déterminer les aspects épidémiologiques, les différentes techniques de fixation chirurgicale des fractures de la patella et les résultats. Patients et Méthodes : il s'est agi d'une étude rétrospective allant de $1^{\text {er }}$ janvier 2013 au 31 décembre 2017 et portant sur les dossiers des patients âgés de 15 ans et plus, traités chirurgicalement pour une fracture de la patella dans notre centre. Résultats : Le travail a concerné 36 patients dont 28 hommes $(77,8 \%)$ et 8 femmes $(22,2 \%)$ avec un âge moyen de 35,9 ans. Les AVP ont constitué la première cause de ces fractures. Les fractures étaient ouvertes dans 44,4\% de cas. Le type II selon Ricard et Moulay était le plus représenté avec $58,3 \%$ des cas. La majorité des patients soit $80,5 \%$ a été prise en charge par haubanage. Cinq (5) cas d'hématomes, une (1) infection post- opératoire, et trois (3) cas de raideur du genou ont compliqué les suites à court et à moyen termes. Avec un recul moyen de 2 ans 6 mois, sur le plan radiologique, la consolidation normale était obtenue dans $86,1 \%$ des cas. Deux (2) cas de retard de consolidation et trois (3) cas de pseudarthroses ont été notés. Trois (3) cas d'arthrose fémoro-patellaire sont apparus à deux ans. Sur le plan fonctionnel, on retrouvait selon le score de Bosman 27,8\% d'excellents, $63,9 \%$ de bons, $8,3 \%$ de mauvais résultats et le haubanage semble donner d'excellents et de bons résultats. Conclusion : Le traitement des fractures transversales de la patella doit être exclusivement chirurgical. Le haubanage constitue une technique d'ostéosynthèse de choix. Néanmoins les complications notamment la raideur du genou, la pseudarthrose et l'arthrose fémoro-patellaire ne sont pas rares.

Mots clés : Fracture patella, haubanage, raideur, arthrose

\section{Introduction}

Les fractures de la patella, relativement rares, représentent environ $1 \%$ des fractures du squelette (NEYRET, 1995). Leur incidence globale serait de 10,7 pour 100000 personnes par an (COURT-BROWN, 2006). Plusieurs travaux s'y rapportent (SAYUM, 2015). 
Elles s'accompagnent souvent d'une rupture de l'appareil extenseur du genou et de lésions cartilagineuses graves qui, en l'absence de traitement, entraînent un déficit permanent et sévère de l'extension (HOHL, 1986). Elles menacent alors la fonction du genou et par conséquent l'avenir socioprofessionnel du traumatisé. Le pronostic de ces fractures dépend de la lésion anatomique et de la qualité de la prise en charge (COUDANE, 1999).

Le traitement des fractures transversales ou déplacées avec perte de congruence articulaire, est souvent chirurgical à foyer ouvert et fait appel à différentes techniques qui doivent permettre de restaurer la continuité de l'appareil extenseur du genou et la mise en route d'une rééducation précoce (MELVIN, 2011).

En raison d'une part du caractère onéreux du traitement chirurgical et d'autre part du manque de moyens financiers des patients qui le plus souvent sont non assurés, ces fractures étaient le plus souvent traités orthopédiquement avec des résultats fonctionnels à la limite, acceptables (ABALO, 2013).

Avec le constat que ces fractures dès lors, sont de plus en plus opérées, nous avons entrepris ce travail dans le but de déterminer les aspects épidémiologiques, les différentes techniques chirurgicales employées et les résultats de ce traitement chirurgical dans notre centre.

\section{Méthodologie}

Dans cette étude rétrospective couvrant la période de janvier 2013 à décembre 2017, les dossiers médicaux de patients des deux sexes ayant été traités chirurgicalement par plusieurs opérateurs, pour fracture de la patella confirmée radiologiquement et revus en contrôle post opératoire dans notre service, ont été examinés.

Les dossiers de patients ayant eu des fractures sur patella malformées, les lésions osseuses associées du genou (fractures de l'extrémité inferieure du fémur et des plateaux tibiaux) et les lésions ligamentaires diagnostiquées du genou n'ont pas été retenus pour l'étude. Les traits de fracture ont été classés selon Ricard et Moulay (RICARD, 1975). Les résultats fonctionnels ont été évalués selon le score clinique de Bosman (BOSMAN, 1981).

\section{Résultats}

Trente-six (36) patients répondaient à nos critères de sélection dont 28 hommes et 8 femmes (sex-ratio $\mathrm{H} / \mathrm{F}$ de 3,5). La fréquence annuelle était de 7,2 patients par an. Leur âge moyen était de 35,9 ans avec les extrêmes de 19 et de 70 ans. Les accidents de circulation routière étaient en cause dans 80,5\%. Il s'agissait de fracture ouverte dans $44,4 \%$. Le tableau 1 indique la caractérisation des fractures selon Ricard et Mouley. 
Tableau 1: distribution selon la classification de Ricard et Mouley

\begin{tabular}{lcc}
\hline & Effectif $(\mathrm{n})$ & Pourcentage $(\%)$ \\
\hline Type 1 & 8 & 22,2 \\
Type 2 & 21 & 58,3 \\
Type 3 & 7 & 19,5 \\
Total & 36 & 100 \\
\hline
\end{tabular}

Les traits de fracture étaient comminutifs dans $77,8 \%$ contre des traits transversaux dans $22,2 \%$. On notait l'existence des lésions osseuses associées dans $50 \%$ des cas, parmi lesquelles les fractures du membre inférieur homolatéral représentaient plus de la moitié (tableau 2).

Tableau 2: distribution des lésions osseuses associées

\begin{tabular}{lcc}
\hline & Effectif (n) & Pourcentage (\%) \\
\hline Traumatisme cranio-encéphalique & 4 & 22,2 \\
Fracture du bassin & 1 & 5,5 \\
$\begin{array}{l}\text { Fracture du membre pelvien } \\
\text { homolatéral }\end{array}$ & 10 & 55,7 \\
$\begin{array}{l}\text { Fracture du membre pelvien } \\
\text { controlatéral }\end{array}$ & 1 & 5,5 \\
$\begin{array}{l}\text { Fracture de membre thoracique } \\
\text { Total }\end{array}$ & 2 & 11,1 \\
\end{tabular}

Le délai moyen de prise en charge était de 23 jours. Suivant les techniques d'ostéosynthèse, vingt-neuf (29) patients soit $80,6 \%$ avaient bénéficié d'un haubanage. Le reste a été traité par embrochage-cerclage. Cinq (5) patients, soit 13,9\% ont présenté en post opératoire immédiat un hématome post- opératoire ayant bien évolué sous traitement par cryothérapie. Un (01) patient $(2,78 \%)$, a présenté une infection secondaire consécutive à la présence d'un corps étranger intra articulaire, noté dans les suites d'une fracture ouverte de la patella. Ce cas a bénéficié d'une reprise chirurgicale et a bien évolué en post opératoire. Trois (3) patients $(8,3 \%)$ ont présenté au tour de trois mois une raideur du genou, ce qui a justifié une mobilisation sous anesthésie générale. Ces cas raideurs étaient survenus dans les suites de fractures ouvertes.

Tableau 3 : résultats fonctionnels globaux

\begin{tabular}{lcc}
\hline & Effectif $(\mathrm{n})$ & Pourcentage $(\%)$ \\
\hline Excellent & 10 & 27,8 \\
Bon & 23 & 63,9 \\
Mauvais & 3 & 8,3 \\
Total & 36 & 100 \\
\hline
\end{tabular}

Au recul moyen de 2 ans 6 mois, 86,1\% ont consolidés normalement avec le traitement chirurgical. Nous avons recensé deux cas $(5,6 \%)$ de retard de consolidation et trois cas $(8,3 \%)$ de pseudarthrose. Nous n'avons pas recensé de cas de cal vicieux. Trois (3) patients $(8,3 \%)$, dont un traité par haubanage et deux (2) par embrochage-cerclage, ont présenté chacun une 
arthrose fémoro-patellaire à 2 ans environ. Cette arthrose était médiale dans les trois (3) cas.

Les résultats fonctionnels globaux selon le score clinique de Bosman sont résumés dans le tableau 3. Le haubanage a donné plus d'excellents et bons résultats fonctionnels (tableau 4 ).

Tableau 4 : résultats fonctionnels selon le type de technique d'ostéosynthèse utilisée

\begin{tabular}{lccccc}
\hline & \multicolumn{2}{c}{ Haubanage } & \multicolumn{2}{c}{ Embrochage-cerclage } & Total \\
& $\mathrm{n}$ & $(\%)$ & $\mathrm{n}$ & $(\%)$ & \\
\hline Excellent & 8 & $(27,6)$ & 2 & $(28,6)$ & 10 \\
Bon & 19 & $(65,5)$ & 4 & $(57,1)$ & 23 \\
Mauvais & 2 & $(6,9)$ & 1 & $(14,3)$ & 3 \\
Total & 29 & $(100)$ & 7 & $(100)$ & 36 \\
\hline
\end{tabular}

\section{Discussion}

Cette étude, en dépit de son caractère rétrospectif, de la petite taille de l'échantillon et son aspect monocentrique a permis de dégager les grandes tendances épidémiologiques, thérapeutiques et évolutifs des fractures de la patella dans notre centre.

Ainsi, dans le volume de nos activités de traumatologie courante, les fractures de la patella avec une incidence de 7,2 cas par an, semblent relativement rares comme l'ont indiqué plusieurs travaux (COURT-BROWN, 2006 ; SAYUM, 2015).

Les accidents de circulation routière ont été les plus grands pourvoyeurs. Ces accidents surviennent le plus souvent sur des motocyclistes dont l'habitude vestimentaire est souvent faite de pantalon en tissu léger protégeant peu la patella en cas de choc direct d'où la proportion relativement élevée $(44,4 \%)$ de lésions ouvertes contre 2 à $7 \%$ dans des endroits géographiques où le jean pantalon est le mode vestimentaire le plus commun (DY, 2012).

Le but du traitement des fractures de la patella est la restitution de la congruence articulaire et la restauration de l'appareil extenseur du genou. De nos jours, la chirurgie est devenue le premier choix dans le traitement des fractures de la patella (SUH, 2018) ; plusieurs méthodes de fixation telles que le cerclage, le haubanage, l'exo fixation et le vissage percutané, ont été suggérées (GARDNER, 2005 ; YOUNG, 2010).

Dans notre étude, la technique chirurgicale la plus utilisée était le haubanage $(80,6 \%)$. Le haubanage constitue un mode d'ostéosynthèse qui trouve son indication de choix dans les fractures transversales déplacées. Il a été plus utilisé dans notre étude non seulement à cause de sa simplicité technique, mais aussi parce qu'il permet de créer une bonne compression du foyer de fracture. Son avantage est qu'il transforme les forces de tractions exercées par le système quadricipital sur la patella en forces de compression, et autorise ainsi une rééducation fonctionnelle précoce. 
Les cas de raideur dans ce travail $(8,3 \%)$, méritent notre attention. Ces raideurs seraient probablement dues au retard dans la prise en charge chirurgical combiné à l'immobilisation prolongée et au retard dans le démarrage de la rééducation. Cependant l'ouverture du foyer de fracture semble être un facteur à prendre en compte dans la survenue de raideur. Une analyse plus fine de ce constat dans les études ultérieure serait intéressante. Selon Caudane H. (COUDANE, 1999), la raideur du genou peut également s'expliquer par un simple cloisonnement post hémarthrosique du cul de sac quadricipital ou par des adhérences du quadriceps accompagnant une fracture diaphysaire du fémur.

Le haubanage a donné des résultats extraordinaires sur la consolidation osseuse. Les cas de retard de consolidation et de pseudarthrose rencontrés chez certains patients pourraient s'expliqués non seulement par l'importance de la comminution des fractures mais aussi par un montage probablement insuffisant du matériel d'ostéosynthèse.

Dans ce travail, le haubanage semble avoir donné plus d'excellents et bons résultats, contrairement à l'embrochage-cerclage qui a donné plus de mauvais résultats. Selon Abdoul Wahab et al. (ABDOUL, 2018), le haubanage a prouvé sa supériorité par rapport aux autres méthodes et constitue un mode d'ostéosynthèse de choix qui trouve son indication dans les fractures transversales simples et déplacées. Dans la méta analyse de Sayum F. (SAYUM, 2015) sur le traitement chirurgical conservateur des fractures de patella de l'adulte, il n'y a aucune preuve d'essais contrôlés randomisés évaluant les résultats des différents types d'ostéosynthèse.

Toutefois les travaux effectués sur la chirurgie ouverte (foyer ouvert) utilisant les implants traditionnels comme les broches, les fils d'acier, les vis sur les fractures transversales indiquent que la méthode de fixation la plus pratiquée est le système de tension (HEUSINKVELD, 2013) comme dans notre étude car la majorité des fractures de la patella est transversale (SUH, 2018). Le reproche fait à ce procédé reste à part la douleur et l'irritation postopératoires liées à la présence de matériel, l'insuffisance de compression pouvant occasionner des déplacements secondaires aux mouvements du genou (CARPENTER, 1997).

\section{Conclusion}

Les fractures de la patella occupent une faible part des activités de traumatologie courante. Elles sont souvent ouvertes et comminutives. La technique de fixation de choix est le haubanage qui semble donner de bons résultats. Cependant, la proportion de raideur suite aux fractures ouvertes n'est pas négligeable. Il convient de réduire les délais de prise en charge et explorer l'impact éventuel de l'ouverture dans la survenue de la raideur, dans les études ultérieures. 


\section{References:}

1. NEYRET P. (1995). Les fractures de la rotule (fractures sur prothèse exceptées), Cahiers d'enseignement de la SOFCOT Paris. Expansion Scientifique Française; 52: 123-135.

2. COURT-BROWN CM, CAESAR B. (2006). Epidemiology of adult fractures: A review. Injury;37(8):691-7.

3. SAYUM FJ, LENZA M, TEIXEIRA DE CARVALHO R, PIRES O, COHEN M, BELLOTI JC. (2015). Interventions for treating fractures of the patella in adults. Cochrane Database Syst Rev. Feb 27;(2):CD009651.

4. HOHL M. (1986). Fractures of the patella. In: C. A. Rockwood Jr and D. P. Green, ed. Fractures, Philadelphia. J. B. Lippingcott; 1975, 11481156 Paris, Edition Masson : 177-181.

5. CAUDANE H, HUTTIN P. (1999). Ruptures de l'appareil extenseur du genou. Encycl. Méd. Chir. (Elsevier, Paris), Appareil locomoteur 14-081-A-10; 12p.

6. MELVIN JS, MEHTA S. (2011). Patellar fractures in adults.

7. J Am Acad Orthop Surg Apr;19(4):198-207.

8. ABALO A, BIRAMAH B, BAKRIGAH B, AMAKOUTOU K, WALLA A, JAMES Y, et al. (2013). Fractures de la Patella: Aspects épidémiologiques, thérapeutiques et évolutifs au CHU SO. J. Rech. Sci. Univ. Lomé (Togo); vol 15, $\mathrm{N}^{\circ} 3$

9. RICARD L, MOULAY A. (1975). Les fractures de la rotule. Cahiers d'enseignement de la SOFCOT. Expansion scientifique française, Edit., Paris ; 1: 75-91.

10. BOSMAN O, KIVILUOTO O, NIRMANO J. (1981). Comminuted displaced fractures of the patella. Injury ; 13: 193-202.

11. DY C, LITTLE M, BERKES M, MA Y, ROBERTS T, HELFET D, et al. (2012). Meta-analysis of re-operation, nonunion, and infection after open reduction and internal fixation of patella fractures. J. of Trauma and Acute Care Surgery ; 73(4):928-32.

12. SUH KT, SUH JD, CHO. (2018). Open reduction and internal fixation of comminuted patellar fractures with headless compression screws and wiring technique. J Orthop Sci. Jan;23(1):97-104.

13. GARDNER MJ, GRIFFITH MH, LAWRENCE BD, LORICH DG. (2005). Complete exposure of the articular surface for fixation of patellar fractures. J Orthop Trauma Feb;19(2):118 23.

14. YANG L, YUEPING O, WEN Y. (2010). Management of displaced comminuted patellar fracture with titanium cable cerclage. Knee Aug;17(4):283 6 . 
15. ABDOUL WAHAB A, ZIRBINE A, KOINI M, BADIO S. (2018). Les Fractures de la Patella: Aspects épidémiologiques, lésionnels, thérapeutiques et évolutifs à propos de 162 Cas.

16. Health Sci. Dis ; 19 (1) : 53-7.

17. HEUSINKVELD M, DEN HAMER A, TRAA W, OOMEN P, MAFFULLI N. (2013). Treatment of transverse patellar fractures: a comparison between metallic and nonmetallic implants.

18. British Medical Bulletin ; 107:69-85.

19. SUH K, SUH J, CHO H. (2018). Open reduction and internal fixation of comminuted patellar fractures with headless compression screws and wiring technique. J Orthop Sci. Jan; 23(1):97-104.

20. CARPENTER JE, KASMAN RA, PATEL N et al. (1997). Biomechanical evaluation of current patella fracture fixation techniques. J. Orthop Trauma ; 11:351-56. 